\title{
Vocalization during copulation behavior in greater horseshoe bats, Rhinolophus ferrumequinum
}

\author{
LIU Ying ${ }^{1,2}$, METZNER Walter $^{2 *} \&$ FENG Jiang ${ }^{1 *}$ \\ ${ }^{1}$ Jilin Key Laboratory of Animal Resource Conservation and Utilization, Northeast Normal University, Changchun 130024, China; \\ ${ }^{2}$ Department of Integrative Biology \& Physiology, University of California, 621 Charles E. Young Dr. S., Box 951606, \\ Los Angeles, CA 90095, USA
}

Received November 20, 2012; accepted February 25, 2013; published online April 8, 2013

\begin{abstract}
Vocal communication plays an important role for individual recognition and male-female interaction during mating in greater horseshoe bats, especially in respect to mate fidelity, which ensures that the bats can maintain a stable social organization. Few studies, however, have addressed the calling behavior during copulating in bats. Here, we initially report the copulation vocalizations and behaviors of both male and female greater horseshoe bats. During copulation, the male assumed a dorsal position and arched his back, arming around the female using his feet and thumbs. The male repeatedly produced very short constant frequency (SCF) syllables with high intensity and repetition rate (male 1: $16.48 \pm 4.8 \mathrm{~ms}$, male $2: 17.79 \pm 4.03 \mathrm{~ms}$ ) when he tried to insert the penis into the female, and then long syllables (male 1: $42.08 \pm 12.67 \mathrm{~ms}$, male $2: 43.02 \pm 11.44 \mathrm{~ms}$ ) after penile insertion. The female bats sometime refused the male bats in the early phase of copulations as emitting noise bursts and broad-band vocalizations, but kept silence during actual copulation. We also found that the SCF copulation calls of one male remained stable peak frequencies on different copulation days although its echolocation call frequency varied each day. Moreover, different male individuals maintained their own "private frequency" in the SCF copulation calls. Therefore, we predicted that the SCF copulation calls may serve as an indicator for female greater horseshoe bats to recognize the mating males in order to maintain mate fidelity because horseshoe bats exhibit sexual segregation before mating. Our results stipulate further studies on mating system and copulation strategies in polygynous bats. Such work may also aid in promoting the preservation of greater horseshoe bats.
\end{abstract}

greater horseshoe bat, copulation, communication calls, mate fidelity

Citation: $\quad$ Liu Y, Metzner W, Feng J. Vocalization during copulation behavior in greater horseshoe bats, Rhinolophus ferrumequinum. Chin Sci Bull, 2013, 58: 2179-2184, doi: 10.1007/s11434-013-5793-3

Studies on a wide range of taxa have documented that kinship plays an important role in the evolution and maintenance of social organization [1-4]. Most sexually reproducing species have to determine the individual to combine gametes with, so that they can keep the individual fitness under strong selection pressure [5,6]. They would select a mate with the appropriate species and sex, as well as appropriate quality and genetic compatibility to reproduce successfully [7]. In order to select potential partners, most sexually reproducing animals use complex communication signals [8-10], because the communication signals carry many

*Corresponding authors (email: fengj@nenu.edu.cn; metzner@ucla.edu) species-specific features such as morphology, courtship postures and degree of sexual size dimorphism [11-13].

Since mate choice may strongly affect the animals' fitness, it would be very important for females to choose the right sexual partner in sexual reproduction $[5,14]$. Therefore, the signals emitted from males for attracting females would be crucial during the mating season [15]. Especially in echolocation bats, vocalization plays a very important role in social recognition, such as in mother-infant reunion [16-18]. Male vocal displays also have been well documented in some bats, such as Saccopteryx bilineata [19], Nyctalus noctula [20] and Pipistrellus pipistrellus [21], indicating that male bats have evolved vocalization to defend territory, 
attract mate, or both. For example, male Pipistrellus nathusii bats have stable aggregations of male roosts, vocalization sites, and complex vocal advertising during the mating season [22].

Successful reproduction often involves complex communication [23]. Previous researches have focused on the advertisement calls of male bats attracting females before copulation [24,25] and on the ways in which individual bats assess the quality of potential mates [26]. However, few studies have been conducted on the calls during the process of copulating in bats, except Barclay and Thomas [27], who studied the copulation vocalizations in Myotis lucifugus [27]. They found that the copulation calls were discrete vocalizations and relatively invariable and distinct in structure from any other vocalization used by this species, and that the copulation calls had tonal syllables with low frequency and rising frequency modulations.

Greater horseshoe bats have been documented to show mate fidelity as well as sexual segregation before mating [3]. Thus, male-female reunion is crucial for the maintenance of social organization in this species. In communication systems of animal, a repertoire of graded signals, each used in a variety of contexts, allows to transfer finely detailed information. Ma et al. [28] reported the greater horseshoe bats have a variety of communication calls with 17 syllable types combined into at least six types of simple phrases and four combination phrases [28]. Therefore, we predict that greater horseshoe bats would produce communication calls with very different spectral and temporal characteristics compared to echolocation calls during copulation. The aim of the present study was to find copulation calls of horseshoe bats and test the hypothesis that bats use "private", i.e. individually distinct communication calls during mating.

\section{Materials and methods}

We kept 29 adult greater horseshoe bats, $R$. ferrumequinum captured from near Beijing, China in two rooms $(5 \mathrm{~m} \times 8 \mathrm{~m} \times$ $3 \mathrm{~m}$ each) with a sliding door for connecting the two rooms at University of California, LA, USA. The rooms had regulated light-dark cycles adjusted with an astronomical light timer to the natural photoperiod for Beijing. The environment of the rooms, as well the feeding methods for keeping the bats has been described elsewhere [28].

The experiments were conducted from January to September, 2008. Each time, we separated two randomly chosen individuals ( 1 male and 1 female, bats gender deter- mined at capture) from the colony and transferred them into an observation cage (wire mesh cage with wire bottom; dimensions $0.6 \mathrm{~m} \times 0.6 \mathrm{~m} \times 1 \mathrm{~m}$; width of mesh $1 \mathrm{~cm} \times 1.5 \mathrm{~cm}$ ). The cage was positioned in the center of one room, separated from the free-flying bats by closing the sliding door [28]. The 2 individuals were kept in the observation cage for up to $12 \mathrm{~h}$ with food and water for calls and behaviors recording. We used nail polish to mark the bats' toe so that we could identify the bats individually. The 13 males and 7 females were used to do the experiments, but only 5 individuals ( 2 males and 3 females) had copulation behaviors.

We used UltraSoundGate 116 (Avisoft Bioacoustics, Berlin, Germany) and Avisoft-SASLab Pro (Avisoft Bioacoustics, version 4.3) for sound recording and analysis, respectively. The frequency response of the condenser microphone (Ultrasoundgate CM16) is between 10 and $150 \mathrm{kHz}$ $( \pm 4 \mathrm{~dB})$. We stored and analyzed sounds on laptop (2 GHz; $80 \mathrm{Mb}$ storage) using a sample rate of $250 \mathrm{kHz}$ at $16 \mathrm{bits} /$ sample. Simultaneously, we also used an infrared closedcircuit camera system (Q-See QS2814C, Digital Peripheral Solutions, Anaheim, CA) to record video of the behaviors of the bats. We set the recording length of each call and each video scene both at $1 \mathrm{~min}$, so that they could match with each other. It allowed us to identify the communication calls indicate which kind of communication behavior without disturbing the bats.

Spectrotemporal features of the calls were analyzed based upon a 1024 pt FFT (Hamming window), with a frequency resolution of $244 \mathrm{~Hz}$ and a temporal resolution of $1.024 \mathrm{~ms}$. Pulse duration (D) and dominant frequency (DF, peak frequency in spectrogram) were used to quantify the spectro-temporal characteristics of communication calls. We measured DF from power spectra and D from oscillograms. All the statistic analysis on data was done using commercial statistics software, SPSS.

\section{Results}

In February and March 2008, we recorded the vocalizations and behaviors of 12 copulations in 5 greater horseshoe bats, 2 males (male 1 and male 2) and 3 females (female 1, female 2 and female 3 ). The pairs and number of copulation behaviors are shown in Table 1 . Every time when we put a male-female pair into the observation cage, the male approached the female and touched the female with one of his wings or his mouth and produced typical echolocation calls with normal durations and interpulse intervals (Table 2).

Table 1 The pairs of mated bats and numbers of copulation behaviors happened on each day

\begin{tabular}{lcccc}
\hline & Day 1 & Day 2 & Day 3 & Day 4 \\
\hline Male 1+female 1 & 3 times & 2 times & 1 time & 2 times \\
Male 1+female 2 & & 1 time & & 1 time \\
Male 2+female 3 & & & & \\
\hline
\end{tabular}




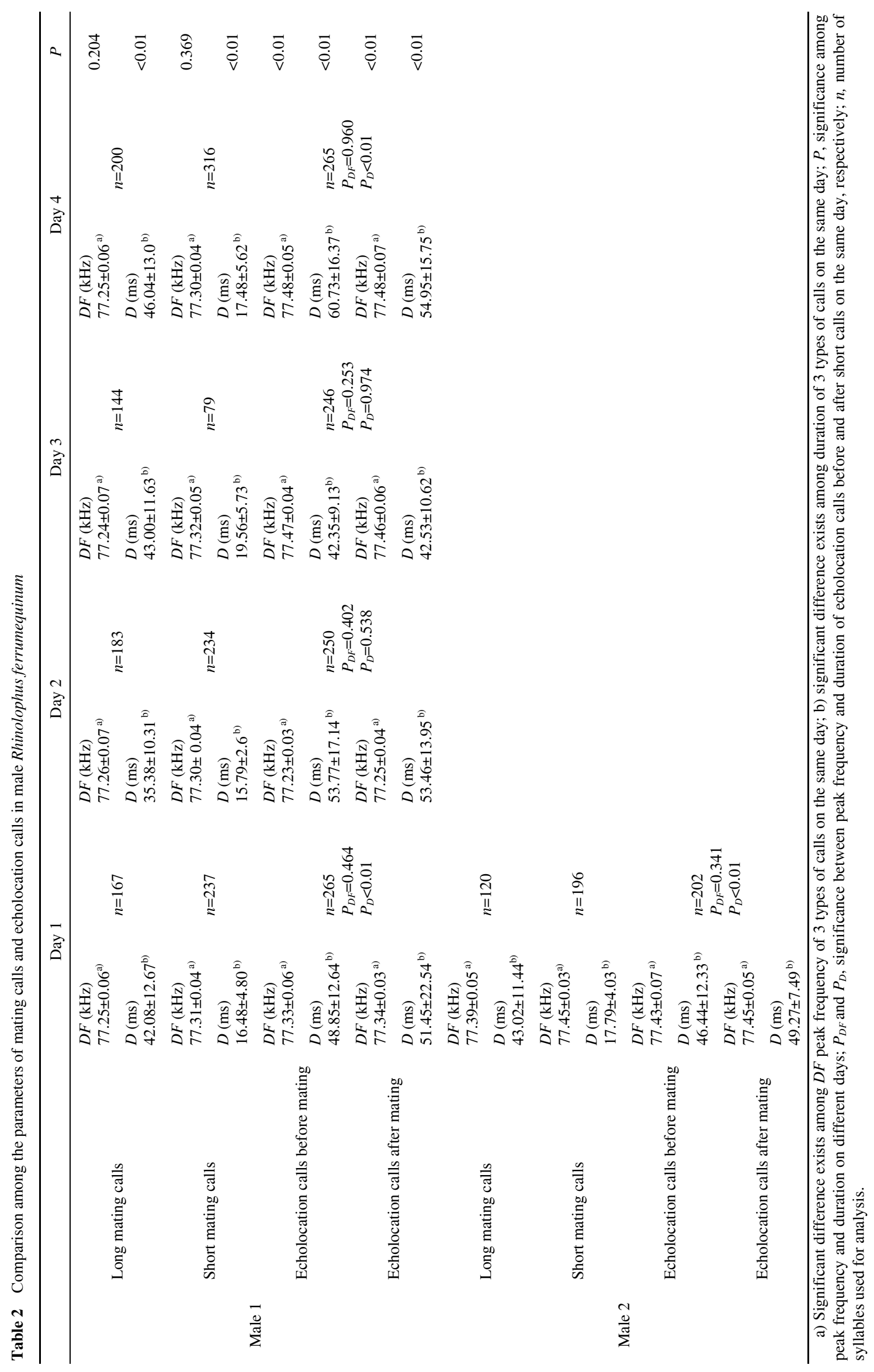


Copulations were initiated by the male immediately after he contacted with a female, but they were not preceded by overt precopulatory interactions. After contact, both the male and female hung on the ceiling of the cage. The male assumed a dorsal position and arched his back. He armed around the female using his feet and thumbs. During the early copulation phase, we observed that the females used wings to flap the males, which seemed like fighting with the male bats in 3 copulations (female 1 in 2 copulations, female 2 in 1 copulation). At other times, however, the females were passive or torpid and did not struggle.

The male repeatedly emitted a "copulation call", including 2 repertoire types, long syllables (male 1: $42.08 \pm 12.67$ $\mathrm{ms}$, male $2: 43.02 \pm 11.44 \mathrm{~ms}$ ) and very short syllables (male 1: $16.48 \pm 4.8 \mathrm{~ms}$, male $2: 17.79 \pm 4.03 \mathrm{~ms}$ ) with high intensity and repetition rate (Figure 1). During each copulation, the male produced more than 10 sequences of copulation calls containing around 15-20 long syllables and 20-30 very short syllables in each sequence. Males emitted very short syllables when they tried to insert the penis into the females, and then longer syllables after insertion. We did not find such courtship calls or behavior in males before copulation. Instead, the male bats only produced normal echolocation calls before and after copulation. We observed male 1 copulate on 4 different days and male 2 copulate on 1 day. Among the 11 copulations over a period of $4 \mathrm{~d}$ in male 1 , the peak frequencies of long copulation calls were constant (Independent-samples $T$ test, $t=2.354, P=0.204$ ), as well as the peak frequency of short copulation calls (Independent-
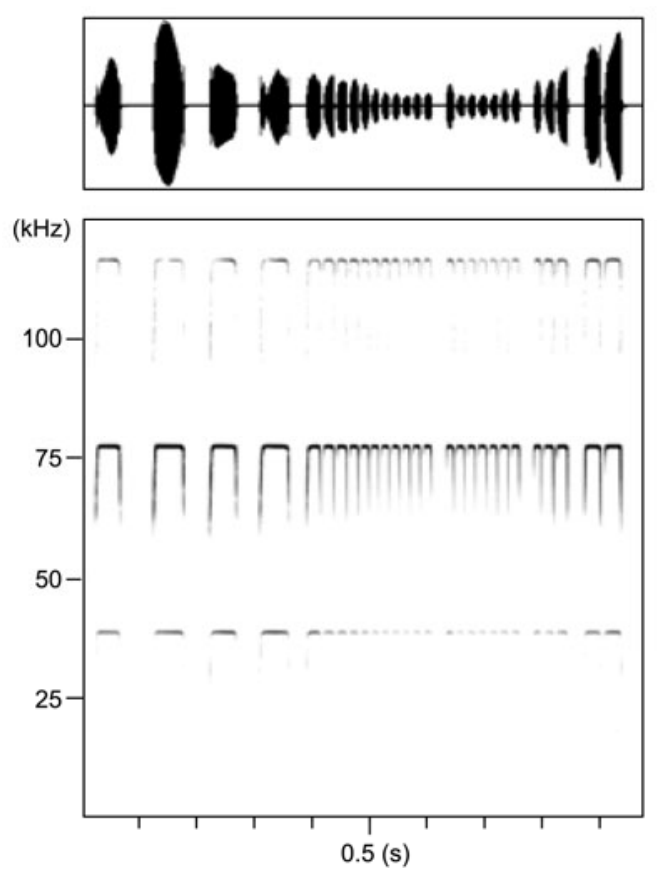

Figure 1 A part of one of the sequences of the communication calls during one copulation of Rhinolophus ferrumequinum, male 1, including the long constant frequency communication syllables and the very short constant frequency communication (SCF) syllables. samples $T$ test, $t=1.630, P=0.369)$. On each copulation day, the peak frequencies and durations of echolocation calls before and after copulation were also constant (Table 2). However, the echolocation calls on different copulation days exhibited significant differences in their peak frequencies (ANOVAs, $F=24.182, P<0.01$ ). Moreover, significant difference (Independent-samples $T$ test, $t=14.249, P<0.01$ ) existed in the peak frequencies of copulation calls between male 1 and male 2, with male 2 having had higher peak frequencies than male 1 (Table 2).

The female bats emitted noise burst and broad-band vocalizations typical of agonistic encounters when they flapped the males in the early phase of copulations, while they kept silent during the copulations. Furthermore, the spectrums of vocalization in female 1 and female 2 were different. Female 1 emitted composite syllables starting with broad-band noise burst components (BNB) and ending with sinusoidally upward frequency-modulated component (sUFM) (Figure 2(a)). Female 2 produced combination phrases constantly contained 2 types of syllables: descending rippled modulatedfrequency syllable (dRFM) (the left one syllable in Figure 2(b)) and noise burst-tonal (NB-tonal) upward modulatedfrequency syllables (the right three syllables in Figure 2(b)). The communication calls in females were classified according to Ma et al. [28] and Kanwal et al. [29].

\section{Discussion}

This study is the first to report vocalizations during copulation of the greater horseshoe bats. It also provides detailed data on bat mating behavior, which is important in the context of bat conservation efforts.

The tonal stereotyped SCF calls produced by male greater horseshoe bats during copulation were different from the rest of their communication repertoire and emitted in different behavioral contexts. As Ma et al. [28] described, the SCF syllables have much shorter duration than the normal duration of echolocation pulses, but there is no gradual transition between normal echolocation pulses and SCF syllables [30,31]. Therefore, the SCF syllable should be communication calls rather than echolocation calls although they seemed similar to extremely short echolocation pulses,

In the present study, male greater horseshoe bats continued to produce copulation calls whether the females struggled or not. Barclay and Thomas [27], however, indicated that in Myotis lucifugus, if the recipient bats did not struggle, the male bats would not emit the copulation call [27]. In contrast, male greater horseshoe bats produced SCF calls only when they tempted to copulate, i.e. tried to insert the penis into females. Other mammals often use visual or tactile precopulatory displays to indicate sexual intention and to synchronize sexual behaviors, such as baboons [32]. But the greater horseshoe bats have none of the displays except vocalizations [33], so the dorsal position of the male and 
(a)
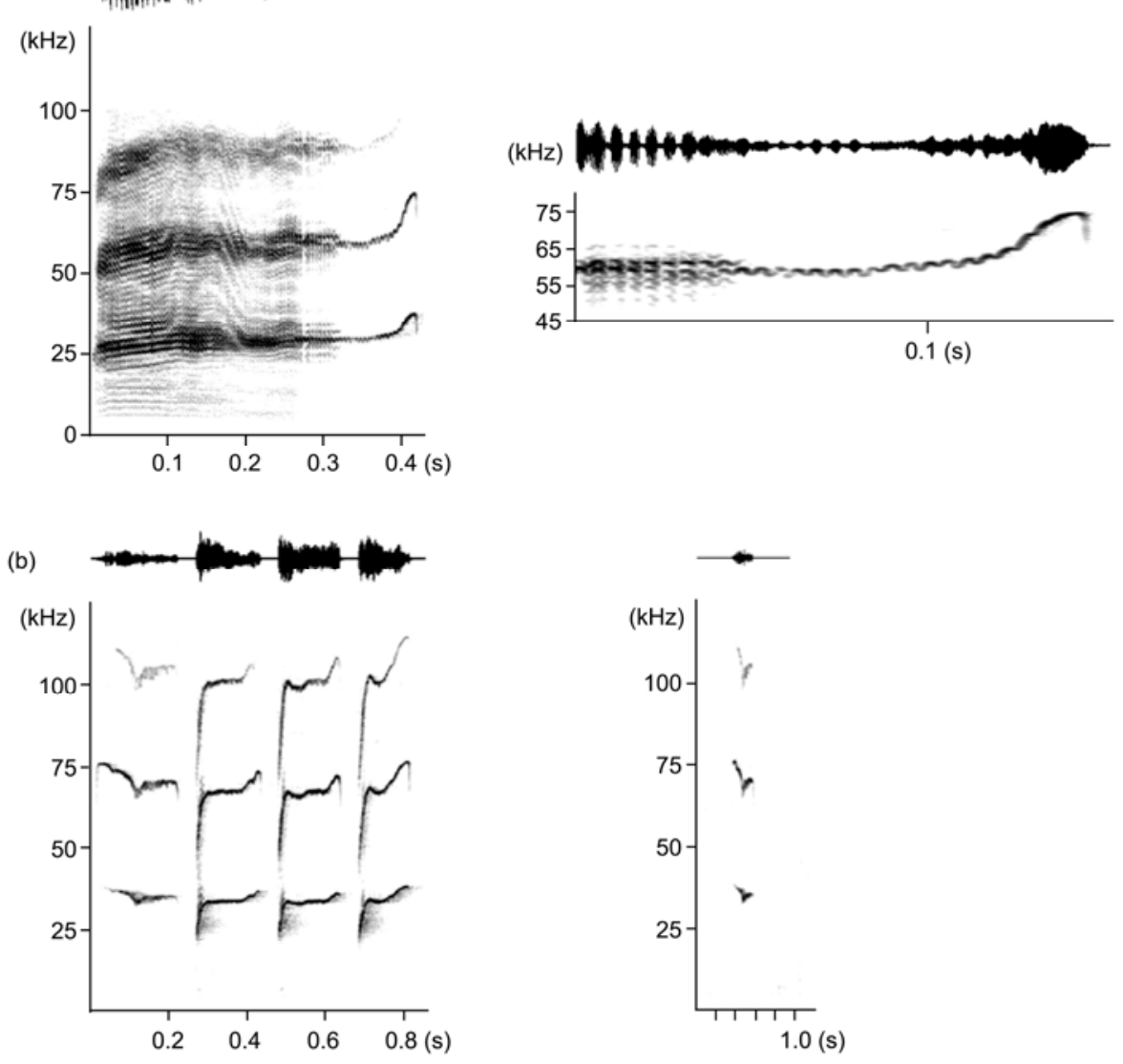

Figure 2 Syllable samples of each communication call of female Rhinolophus ferrumequinum in the early copulation phrase. (a) Composite syllable with BNB and sUFM of female 1, the sUFM part of the second harmonic component with higher spectrotemporal resolution shown at the right; (b) combination phrase of female 2 including one descending rippled modulated-frequency (dRFM) syllable (left) and three noise burst-tonal (NB-tonal) upward modulated-frequency syllables (right), and the zoomed out spectrum of dRFM was shown at the right.

restraining of the female might be misconstrued by the females as aggressive rather than sexual behavior, especially in the dense groups of bats within roosts. The pure SCF calls have very short durations and interpulse intervals, as well the clear peak frequency and amplitude, which would be helpful to convey sexual intent in a rapid and unambiguous way, thereby promoting the close contact required for mating. Thus, the vocalizations during copulation might carry information used for appeasing females and convey the male's sexual rather than aggressive motivation.

We found that the 2 males have private SCF calls with different peak frequency (Table 2). Furthermore, in male 1 the frequencies of echolocation calls varied from day to day, sometimes by as much as $240 \mathrm{~Hz}$, whereas SCF frequencies remained remarkably stable ranging from 77.27 to 77.36 $\mathrm{kHz}$ through the 4 copulation days. However, in contrast to the frequency values of echolocation calls and SCF calls, the durations of both call types varied randomly, i.e. the bats have neither stable nor private duration. The results suggested that greater horseshoe bats may use individually distinct, "private" call frequencies for intra-individual communication and recognition. Studies have documented that a "private bandwidth of frequency" may have crucial functions in social communication and sexual behavior, for example, allowing bats to recognize relative individuals or attract copulation partners of right species [34]. In mating season, the male greater horseshoe bats have a polygynous mating system and male territoriality [35]. Thus the malefemale reunion would be necessary for the successful copulation. Previous studies have documented that bats have various reunion cues, such as olfactory cues for sex discrimination and roostmate recognition in Mops condylurus and Chaerephon pumilus [36], chemical cues for colonymate recognition in Eptesicus fuscus [37]. To our present knowledge, no other cues for individual recognition except vocalization cues for mother-infant reunion [16] were reported in greater horseshoe bats. Therefore, we predicted that the individual unique calls of the two male bats in present study may be one helpful cue for the males to be recognized by the females for a successful copulation. However, the prediction still should be further identified in the future studies because in present study it was only derived from the intention showed in the data of 1 individual.

One may argue that our observation on only 5 individuals 
is somewhat limited to determine the characters of copulation calls in greater horseshoe bats. However, male 1 copulated for many times on 4 different days and all produced similar calls, and although male 2 copulated only on one day, he also produced copulation calls with similar spectrum including long and short types to those emitted by male 1. Furthermore, in previous studies, we found that greater horseshoe bats emit constant SCF calls potentially carrying an "individual frequency signature" not only in the context of copulation but also in other behavioral contexts. The SCF calls and echolocation call may be controlled by different auditory feedback (unpublished data). Therefore our conclusion that greater horseshoe bats produced tonal stereotyped calls including both very short and long syllables during copulation is most likely a generally valid observation.

This work was supported by the National Natural Science Foundation of China (31030011 and 30900166), the Ministry of Education of China (MS2011DBSF023), and Jilin Provincial Science and Technology Department (20100148) and NIH grant to Metzner Walter (dc5400).

1 Reeve H K, Westneat D F, Noon W A, et al. DNA fingerprinting reveals high levels of inbreeding in colonies of the eusocial naked mole-rat. Proc Natl Acad Sci USA, 1990, 87: 2496-2500

2 Chapais B, Berman C M. Kinship and Behavior in Primates. Oxford: Oxford University Press, 2004

3 Rossiter S J, Ransome R K, Faulkes C G, et al. Mate fidelity and intralineage polygyny in greater horseshoe bats. Nature, 2005, 437: 408-411

4 Clutton-Brock T H, Lukas D. The evolution of social philopatry and dispersal in female mammals. Mol Ecol, 2012, 21: 472-492

5 Andersson M. Sexual Selection. Princeton: Princeton University Press, 1994

6 Kraaijeveld K, Kraaijeveld-Smit F J L, Maan M E. Sexual selection and speciation: The comparative evidence revisited. Biol Rev, 2011, 86: $367-377$

7 Andersson M, Simmons L. Sexual selection and mate choice. Trends Ecol Evol, 2006, 21: 296-302

8 Searcy W A, Andersson M. Sexual selection and the evolution of song. Ann Rev Ecol Syst, 1986, 17: 507-533

9 Greenspan R J, Ferveur J F. Courtship in Drosophila. Ann Rev Genetics, 2000, 34: 205-232

10 Endler J A, Westcott D A, Madden J R, et al. Animal visual sysytems and the evolution of color patterns: Sensory processing illuminates signal evolution. Evolution, 2005, 59 : 1795-1818

11 Ohtsuka S, Huys R. Sexual dimorphism in calanoid copepods: Morphology and function. Hydrobiologia, 2001, 453/454: 441-466

12 Vanhooydonck B, Herrel A, Damme R. The relationship between dewlap size and performance changes with age and sex in a Green Anole (Anolis carolinensis) lizard population. Behav Ecol Sociobiol, 2005, 59: 157-165

13 Rughetti M, Festa-Bianchet. Seasonal changes in sexual size dimorphism in northern chamois. J Zool, 2011, 28: 257-264

14 Ramm S A, Khoo L, Stockley P. Sexual selection and the rodent baculum: An intraspecific study in the house mouse (Mus musculus domesticus). Genetica, 2010, 138: 129-137

15 McCracken G F, Wilkinson G S. Bat mating systems. In: Crichton E
G, Krutzsch P H, eds. Reproductive Biology of Bats. New York: Academic Press, 2000. 321-362

16 Matsumura S. Mother-infant communication in a horseshoe bat (Rhinolophus ferrumequinum nippon): Development of vocalization. J Mammal, 1979, 60: 76-84

17 Matsumura S. Mother-infant communication in a horseshoe bat (Rhinolophus ferrumequinum nippon): Vocal communication in threeweek-old infants. J Mammal, 1981, 62: 20-28

18 Liu Y, Feng J, Jiang Y L. Vocalization development of greater horseshoe bat, Rhinolophus ferrumequinum (Rhinolophidae, Chiroptera). Folia Zool, 2007, 56: 126-136

19 Davidson S M, Wilkinson G S. Function of male song in the greater white-lined bat, Saccopteryx bilineata. Anim Behav, 2004, 67: 883891

20 Sluiter J W, van Heerdt P F. Seasonal habits of the noctule bat Nyctalus noctula. Arch Nierlandaise Zool, 1966, 16: 432-439

21 Barlow K E, Jones G. Function of pipistrelle social calls: Field data and a playback experiment. Anim Behav, 1997, 53: 991-999

22 Jahelková H, Horáček I. Mating system of a migratory bat, Nathusius' pipistrelle (Pipistrellus nathusii): Different male strategies. Acta Chiropterol, 2011, 13: 123-137

23 Tibbetts E A, Dale J. Individual recognition: It is good to be different. Trends Ecol Evol, 2007, 22: 529-537

24 Lundberg K, Gerell R. Territorial advertisement and mate attraction in the bat, Pipistrellus pipistrellus. Ethology, 1986, 71: 115-124

25 Melendez K V, Feng A S. Communication calls of little brown bats display individual-specific characteristics. J Acoustic Soc Amer, 2010, 128: 919-923

26 Behr O, Helversen O. Bat serenades-complex courtship songs of the sac-winged bat (Saccopteryx bilineata). Behav Ecol Sociobiol, 2004, 56: 106-115

27 Barclay R M R, Thomas D W. Copulation call of Myotis lucifugus: A discrete situation-specific communication signal. J Mammal, 1979, 60: 632-634

28 Ma J, Kobayasi K, Zhang S Y, et al. Vocal communication in adult greater horseshoe bats, Rhinolophus ferrumequinum. J Comp Physiol A, 2006, 192: 535-550

29 Kanwal J S, Matsumura S, Ohlemiller K, et al. Analysis of acoustic elements and syntax in communication sounds emitted by mustached bats. J Acoustic Soc Amer, 1994, 96: 1229-1253

30 Neuweiler G, Metzner W, Heilmann U, et al. Foraging behaviour and echolocation in the rufous horseshoe bat (Rhinolophus rouxi) of Sri Lanka. Behav Ecol Sociobiol, 1987, 20: 53-67

31 Tian B, Schnitzler H U. Echolocation signals of the Greater Horseshoe bat (Rhinolophus ferrumequinum) in transfer flight and during landing. J Acoustical Soc Amer, 1997, 101: 2347-2364

32 Semple S. Individuality and male discrimination of female copulation calls in the yellow baboon. Anim Behav, 2001, 61: 1023-1028

33 Andrews M M, Andrews P T. Ultrasound social calls made by greater horseshoe bats (Rhinolophus ferrumequinum) in a nursery roost. Acta Chiropterol, 2003, 5: 221-234

34 Russo D, Mucedda M, Bello M, et al. Divergent echolocation call frequencies in insular rhinolophids (Chiroptera): A case of character displacement? J Biogeogr, 2007, 34: 2129-2138

35 Rossiter S J, Zubaid A, Mohd-Adnan A, et al. Social organization and genetic structure: Insights from codistributed bat populations. Mol Ecol, 2012, 21: 647-661

36 Bouchard S. Sex discrimination and roostmate recognition by olfactory cues in the African bats, Mops condylurus and Chaerephon pumilus (Chiroptera: Molossidae). J Zool Lond, 2001, 254: 109-117

37 Bloss O, Acree T E, Bolss J M, et al. Potential use of chemical cues for colony-mate recognition in the big brown bat, Eptesicus fuscus. $\mathrm{J}$ Chem Ecol, 2002, 28: 819-834

Open Access This article is distributed under the terms of the Creative Commons Attribution License which permits any use, distribution, and reproduction in any medium, provided the original author(s) and source are credited. 